\title{
A P System Based Model of an Ecosystem of Some Scavenger Birds
}

\author{
Mónica Cardona ${ }^{1}$, M. Angels Colomer ${ }^{1}$, \\ Antoni Margalida ${ }^{4}$, Ignacio Pérez-Hurtado ${ }^{2}$, \\ Mario J. Pérez-Jiménez ${ }^{2}$, Delfí Sanuy ${ }^{3}$, \\ 1 Dpt. of Mathematics, University of Lleida \\ Av. Alcalde Rovira Roure, 191. 25198 Lleida, Spain \\ \{mcardona, colomer\}@matematica.udl.es \\ 2 Research Group on Natural Computing \\ Dpt. of Computer Science and Artificial Intelligence, University of Sevilla \\ Avda. Reina Mercedes s/n, 41012 Sevilla, Spain \\ \{perezh, marper\}@us.es \\ 3 Dpt. of Animal Production, University of Lleida \\ Av. Alcalde Rovira Roure, 191. 25198 Lleida, Spain \\ dsanuy@prodan.udl. cat \\ 4 Bearded Vulture Study \& Protection Group \\ Adpo. 43 E-25520 El Pont de Suert (Lleida), Spain \\ margalida@inf.entorno.es
}

Summary. The Bearded Vulture (Gypaetus Barbatus) is an endangered species in Europe that feeds almost exclusively on bone remains provided by wild and domestic ungulates. In [1], we presented a $\mathrm{P}$ system in order to study the evolution of these species in the Pyrenees (NE Spain). Here, we present a new model that overcomes some limitations of the previous work incorporating other scavenger species (predatory) and additional prey species that provide food for the scavenger intraguild and interact with the Bearded Vulture in the ecosystem. After the validation, the new model can be a useful tool for the study of the evolution and management of the ecosystem. $\mathrm{P}$ systems provide a high level computational modelling framework which integrates the structural and dynamical aspects of ecosystems in a compressive and relevant way. The inherent randomness and uncertainty in ecosystems is captured by using probabilistic strategies.

\section{Introduction}

Since nature is very complex, the perfect model that explains it will be complex too. A complex model is not practical or good to use, so we should obtain a simple and useful model that keeps the most important natural factors. 
The $\mathrm{P}$ system presented in [1] gives good results in order to study the evolution of the ecosystem based on the Bearded Vulture in the Catalan Pyrenees in the short term, but it does not take into account neither important factors such as the population density or the feeding limitations, nor other species that coexist and compete for space and feeding with the Bearded Vulture. Besides, it was accepted at the said model that the population growth rate of the Bearded Vulture was constant.

In the Catalan Pyrenees, in the North-east of Spain, three vulture species inhabits sharing the geographic space and the existent food resources. In this work, we present a $\mathrm{P}$ system for modelling an ecosystem based on three vulture species and the prey species present from which scavengers obtain their food from. Apart from adding two new predator species (the Egyptian Vulture Neophron percnopterus and Eurasian Griffon Vulture Gyps fulvus), we introduce new prey species (making a total of 13 species in comparison to the 5 species appearing at [1]) in the new model that provide feeding resources for the scavenger community. Besides, new rules are introduced to limit the maximum amount of animals that can be supported by the ecosystem as well as the amount of grass available for the herbivorous species. At the new model, it is considered that the population growth rate of the Bearded Vulture varies depending on the surface and orography of the system as well as on existing population. For a good management of the ecosystem, it is suitable to know the biomass every species leaves annually. For this reason, it is interesting to codify this information at the system output.

For the modelling of the ecosystem, we need the biological parameters that are show in the Table 1 . These parameters are obtained experimentally and they quantify the biological basic processes of the species and the physical environment of the ecosystem. The processes modelled are reproduction, feeding and mortality, and the physical factors that have been considered are the geographical limitations.

It has been developed simulator of the model written in JAVA by using the specification language P-Lingua [4]. This simulator allows us to experimentally validate the model as well as study the ecosystem dynamics under different initial conditions.

The paper is organized as follows. Next section shows a formal framework to model ecosystems by means of probabilistic $\mathrm{P}$ systems, and a $\mathrm{P}$ system modelling of the above mentioned ecosystem is presented. In Section 3, we experimentally validate the model presented in this paper by using a P-lingua simulator [4] and we also compare it to the one presented in [1].

\section{A Formal Framework to Model Ecosystems}

In this section, we present a model of the ecosystem described above by means of probabilistic P systems. 
First, we define the $\mathrm{P}$ systems based framework (probabilistic $\mathrm{P}$ systems), where additional features such as electrical charges which describe specific properties in a better way, are used.

Definition 1. A probabilistic $P$ system of degree $q$ is a tuple

$$
\Pi=\left(\Gamma, \mu, \mathcal{M}_{1}, \ldots, \mathcal{M}_{q}, R,\left\{c_{r}\right\}_{r \in R}\right)
$$

where:

- $\Gamma$ is the alphabet (finite and nonempty) of objects (the working alphabet);

- $\mu$ is a membrane structure (a rooted tree), consisting of q membranes, labelled by $1,2, \ldots, q$. The skin membrane is labelled by 1 . We also associate electrical charges with membranes from the set $\{0,+,-\}$, neutral, positive and negative;

- $\mathcal{M}_{1}, \ldots, \mathcal{M}_{q}$ are strings over $\Gamma$, describing the multisets of objects initially placed in the $q$ regions of $\mu$;

- $R$ is a finite set of evolution rules. An evolution rule associated with the membrane labelled by $i$ is of the form

$$
r: u[v]_{i}^{\alpha} \stackrel{c_{r}}{\longrightarrow} u^{\prime}\left[v^{\prime}\right]_{i}^{\alpha^{\prime}}
$$

where $u, v, u^{\prime}, v^{\prime}$ are multisets over $\Gamma, \alpha, \alpha^{\prime} \in\{0,+,-\}, 1 \leq i \leq q$, and $c_{r}$ is a real number between 0 and 1 . Besides, if $r_{1}, \ldots, r_{t}$ are rules whose left-hand side is $u[v]_{i}^{\alpha}$ then it must verify $\sum_{j=1}^{t} c_{r_{j}}=1$, being $c_{r_{j}}$ the probabilistic constant associated with rule $r_{j}$.

We denote by $\left[v \stackrel{c_{r}}{\longrightarrow} v^{\prime}\right]_{i}^{\alpha}$ rule $u[v]_{i}^{\alpha} \stackrel{c_{r}}{\longrightarrow} u^{\prime}\left[v^{\prime}\right]_{i}^{\alpha^{\prime}}$ in the case $u=u^{\prime}=\lambda$, and $\alpha=\alpha^{\prime}$. In the same way, we denote by $u[v]_{i}^{\alpha} \rightarrow u^{\prime}\left[v^{\prime}\right]_{i}^{\alpha^{\prime}}$ rule $u[v]_{i}^{\alpha} \stackrel{c_{r}}{\longrightarrow} u^{\prime}\left[v^{\prime}\right]_{i}^{\alpha^{\prime}}$ in the case $c_{r}=1$.

We assume that a global clock exists, marking the time for the whole system (for all compartments of the system); that is, all membranes and the application of all the rules are synchronized.

The multisets of objects present at any moment in the $n$ regions of the system constitute the configuration of the system at that moment. Particularly, tuple $\left(\mathcal{M}_{1}, \ldots, \mathcal{M}_{q}\right)$ is the initial configuration of the system.

The $\mathrm{P}$ system can pass from one configuration to another by using rules from $R$ as follows:

- A rule $u[v]_{i}^{\alpha} \stackrel{c_{r}}{\longrightarrow} u^{\prime}\left[v^{\prime}\right]_{i}^{\alpha^{\prime}}$ is applicable (with a probability $c_{r}$ ) to a membrane labelled by $i$, and with $\alpha$ as electrical charge, when multiset $u$ is contained in the father of membrane $i$, and multiset $v$ is contained in membrane $i$. When rule $u[v]_{i}^{\alpha} \stackrel{c_{r}}{\longrightarrow} u^{\prime}\left[v^{\prime}\right]_{i}^{\alpha^{\prime}}$ is applied, multiset $u$ (resp. $v$ ) in the father of membrane $i$ (resp. membrane $i$ ) is removed from that membrane and multiset $u^{\prime}$ (resp. $\left.\mathrm{v}^{\prime}\right)$ is produced in it. 
- The rules are applied in a maximal consistent parallelism, that is, all those rules of type $u_{1}\left[v_{1}\right]_{i}^{\alpha} \stackrel{c_{r}}{\longrightarrow} u_{1}^{\prime}\left[v_{1}^{\prime}\right]_{i}^{\alpha^{\prime}}$ and $u_{2}\left[v_{2}\right]_{i}^{\alpha} \stackrel{c_{s}}{\longrightarrow} u_{2}^{\prime}\left[v_{2}^{\prime}\right]_{i}^{\alpha^{\prime}}$ must be applied simultaneously in a maximal way.

- The constant $c_{r}$ associated with rule $r$ indicates the affinity of the said rule for its application.

\subsection{A P System Based Model of the Ecosystem}

Let $D$ be a natural number higher than 0 , which will represent the number of years to be simulated in the evolution of the ecosystem. At the definition of a probabilistic $\mathrm{P}$ system modelling the ecosystem described at Section $1, n=17$ represents the different types of animals of the 13 species which compose the ecosystem under study. We considerer two types of animals for the Red Deer due to the fact that males are very estimated by hunters and this implies the mortality rate of males $(i=6)$ to be higher than that of females $(i=5)$. We also consider two types of animals, denoted by A (annual) and P (periodical), for domestic ones (except for horses) because some of them spend only six months in the mountain.

Next, we present a list of the constants associated with the rules where the corresponding meanings are specified (index $i$ represents the type of animal).

- $g_{i, 1}: 1$ for wild animals and 0 for domestic animals.

- $g_{i, 2}$ : proportion of time they remain in the mountain during the year.

- $g_{i, 3}$ : age at which adult size is reached. This is the age at which the animal eats like and adult does, and at which if the animal dies, the amount of biomass it leaves is similar to the total one left by an adult. Moreover, at this age it will have surpassed the critical early phase during which the mortality rate is high.

- $g_{i, 4}$ : age at which it starts to be fertile.

- $g_{i, 5}$ : age at which it stops being fertile.

- $g_{i, 6}:$ average life expectancy in the ecosystem.

- $g_{i, 7}$ : maximum density of the ecosystem.

- $g_{i, 8}$ : number of animals that survive after reaching maximum density of the ecosystem.

- $k_{i, 1}$ : proportion of females in the population (per one).

- $k_{i, 2}$ : fertility rate (proportion of fertile females that reproduce).

- $k_{i, 3}$ : number of descendants per each fertile female that reproduces.

- $k_{i, 4}$ : it is equal to 0 when the species go through a natural growth and it is equal to 1 when animals are nomadic (the Bearded Vulture moves from one place to another until it is $6-7$ years old, when it settles down).

- $k_{i, 5}$ : population growth (per one).

- $m_{i, 1}$ : natural mortality rate in the first years, age $<g_{i, 3}$ (per one). 
- $m_{i, 2}$ : mortality rate in adult animals, age $\geq g_{i, 3}$ (per one).

- $m_{i, 3}$ : percentage of domestic animals belonging to non-stabilized populations which are withdrawn in the first years.

- $m_{i, 4}$ : is equal to 1 if the animal dies at the age of $g_{i, 6}$ and it is not retired, and it is equal to 0 if the animal does not die at the age of $g_{i, 6}$ but it is retired from the ecosystem.

- $f_{i, 1}$ : amount of bones from young animals when they die, age $<g_{i, 3}$.

- $f_{i, 2}$ : amount of meat from young animals when they die, age $<g_{i, 3}$.

- $f_{i, 3}$ : amount of bones from adult animals when they die, age $\geq g_{i, 3}$.

- $f_{i, 4}$ : amount of meat from adult animals when they die, age $\geq g_{i, 3}$.

- $f_{i, 5}$ : amount of bones necessary per year and animal (1 unit is equal $0.5 \mathrm{~kg}$ of bones).

- $f_{i, 6}$ : amount of grass necessary per year and animal.

- $f_{i, 7}$ : amount of meat necessary per year and animal.

The values of these constants appears at Table 1 and they have been obtained experimentally, except for $k_{i, 4}$ and $m_{i, 4}$ (see [2], [3], [6], [5] for details). Constants $k$ are associated with reproduction rules, constants $m$ are associated with mortality rules, constants $f$ are associated with feeding rules and constants $g$ are associated to the remaining rules.

It is important to highlight that the information associated with an animal is assumed to be referred to units of individuals, except for scavenger birds which are assumed to be referred to pairs.

Let us consider the following probabilistic $\mathrm{P}$ system of degree 2 with (only) two electrical charges (neutral and positive)

$$
\Pi_{D}=\left(\Gamma, \mu, \mathcal{M}_{1}, \mathcal{M}_{2}, R,\left\{c_{r}\right\}_{r \in R}\right)
$$

where:

- $\Gamma=\left\{X_{i j}, Y_{i j}, V_{i j}, Z_{i j}: 1 \leq i \leq n, 0 \leq j \leq g_{i, 6}\right\} \cup$

$\left\{B, G, M, B^{\prime}, G^{\prime}, M^{\prime}, C, C^{\prime}\right\} \cup\left\{h_{s}: 1 \leq s\right\} \cup$

$\left\{H_{i}, H_{i}^{\prime}, F_{i}, F_{i}^{\prime}, T_{i}, a_{i}, b_{0 i}, b_{i}, d_{i}, e_{i}: 1 \leq i \leq n\right\}$

is the working alphabet.

Symbols $X, Y, V$ and $Z$ represent the same animal but in different states. Index $i$ is associated with the type of animal, index $j$ is associated with their age, and $g_{i, 6}$ is the average life expectancy. It also contains the auxiliary symbols $B, B^{\prime}$, which represent bones, $M, M^{\prime}$, which represent meat and $G, G^{\prime}$, which represent the amount of grass available for the feeding of the animals in the ecosystem. Objects $H_{i}, H_{i}^{\prime}$ represent the biomass of bones, and objects $F_{i}, F_{i}^{\prime}$ represent the biomass of meat left by species $i$ in different states. Object $C$ enables the creation of objects $B^{\prime}, M^{\prime}$ and $G^{\prime}$ which codify bones and meat (artificially added by human beings) as well as the grass generated by the 
Table 1: Constants used in the $\mathrm{P}$ system based model

\begin{tabular}{|l|c|c|c|c|c|c|c|c|c|c|c|c|c|}
\hline Specie & $i$ & $g_{i, 1}$ & $g_{i, 2}$ & $g_{i, 3}$ & $g_{i, 4}$ & $g_{i, 5}$ & $g_{i, 6}$ & $g_{i, 7}$ & $g_{i, 8}$ & $k_{i, 1}$ & $k_{i, 2}$ & $k_{i, 3}$ & $k_{i, 4}$ \\
\hline Bearded Vulture & 1 & 1 & 1 & 1 & 8 & 20 & 21 & 60 & 60 & 0.5 & 0.08 & 1 & 1 \\
\hline Egyptian Vulture & 2 & 1 & 0.5 & 1 & 5 & 24 & 25 & 80 & 80 & 0.5 & 0.593 & 1 & 0 \\
\hline Griffon Vulture & 3 & 1 & 1 & 1 & 5 & 24 & 25 & 700 & 700 & 0.5 & 0.75 & 1 & 0 \\
\hline P. chamois & 4 & 1 & 1 & 1 & 2 & 18 & 18 & 15000 & 7500 & 0.55 & 0.75 & 1 & 0 \\
\hline Red deer female & 5 & 1 & 1 & 1 & 2 & 17 & 17 & 4615 & 3230 & 1 & 0.75 & 1 & 0 \\
\hline Red deer male & 6 & 1 & 1 & 1 & 2 & 20 & 20 & 2885 & 2020 & 0 & 0 & 0 & 0 \\
\hline Fallow deer & 7 & 1 & 1 & 1 & 2 & 12 & 12 & 3000 & 2400 & 0.75 & 0.55 & 1 & 0 \\
\hline Roe deer & 8 & 1 & 1 & 1 & 1 & 10 & 10 & 15000 & 7500 & 0.67 & 1 & 1 & 0 \\
\hline Ovis Orientalis & 9 & 1 & 1 & 1 & 2 & 12 & 12 & 1000 & 1000 & 0,5 & 0,9 & 2 & 0 \\
\hline Wild board & 10 & 1 & 1 & 1 & 1 & 4 & 6 & 200000 & 200000 & 0,5 & 0,75 & 4 & 0 \\
\hline Sheep A & 11 & 0 & 1 & 1 & 2 & 8 & 8 & 200000 & 200000 & 0.96 & 0.75 & 1 & 0 \\
\hline Sheep P & 12 & 0 & 0.5 & 1 & 2 & 8 & 8 & 50000 & 50000 & 0.96 & 0.75 & 1 & 0 \\
\hline Bovine A & 13 & 0 & 1 & 2 & 2 & 9 & 14 & 168500 & 168500 & 0.9 & 0.9 & 1 & 0 \\
\hline Bovine P & 14 & 0 & 0.5 & 2 & 2 & 9 & 14 & 168500 & 168500 & 0.9 & 0.9 & 1 & 0 \\
\hline Goat A & 15 & 0 & 1 & 1 & 2 & 8 & 8 & 17000 & 17000 & 0.97 & 0.9 & 1 & 0 \\
\hline Goat P & 16 & 0 & 0.5 & 1 & 2 & 8 & 8 & 17000 & 17000 & 0.97 & 0.9 & 1 & 0 \\
\hline Horse & 17 & 0 & 1 & 3 & 3 & 9 & 20 & 6600 & 6600 & 0.97 & 0.9 & 1 & 0 \\
\hline
\end{tabular}

\begin{tabular}{|l|c|c|c|c|c|c|c|c|c|c|c|c|}
\hline Specie & $i$ & $m_{i, 1}$ & $m_{i, 2}$ & $m_{i, 3}$ & $m_{i, 4}$ & $f_{i, 1}$ & $f_{i, 2}$ & $f_{i, 3}$ & $f_{i, 4}$ & $f_{i, 5}$ & $f_{i, 6}$ & $f_{i, 7}$ \\
\hline Bearded Vulture & 1 & 0.06 & 0.12 & 0 & 1 & 0 & 0 & 0 & 0 & 920 & 0 & 0 \\
\hline Egyptian Vulture & 2 & 0.17 & 0.07 & 0 & 1 & 0 & 0 & 0 & 0 & 0 & 0 & 332 \\
\hline Griffon Vulture & 3 & 0.03 & 0.01 & 0 & 1 & 0 & 0 & 0 & 0 & 0 & 0 & 800 \\
\hline P. chamois & 4 & 0.6 & 0.06 & 0 & 1 & 6 & 8 & 12 & 48 & 0 & 550 & 0 \\
\hline Red deer female & 5 & 0.34 & 0.06 & 0 & 1 & 15 & 26 & 30 & 120 & 0 & 2540 & 0 \\
\hline Red deer male & 6 & 0.34 & 0.36 & 0 & 1 & 24 & 30 & 48 & 192 & 0 & 2540 & 0 \\
\hline Fallow deer & 7 & 0.5 & 0.06 & 0 & 1 & 2 & 28 & 4 & 74 & 0 & 1100 & 0 \\
\hline Roe deer & 8 & 0.58 & 0.06 & 0 & 1 & 1 & 8 & 2 & 38 & 0 & 600 & 0 \\
\hline Ovis Orientalis & 9 & 0,6 & 0,06 & 0 & 1 & 7 & 8 & 12 & 44 & 0 & 550 & 0 \\
\hline Wild board & 10 & 0,69 & 0,35 & 0 & 1 & 8 & 12 & 24 & 120 & 0 & 730 & 0 \\
\hline Sheep A & 11 & 0.15 & 0.030 & 0.59 & 0 & 7 & 8 & 14 & 56 & 0 & 1320 & 0 \\
\hline Sheep P & 12 & 0.15 & 0.030 & 0.59 & 0 & 7 & 8 & 14 & 56 & 0 & 1320 & 0 \\
\hline Bovine A & 13 & 0.057 & 0.045 & 0 & 0 & 21 & 119 & 12 & 1038 & 0 & 11000 & 0 \\
\hline Bovine P & 14 & 0.057 & 0.045 & 0 & 0 & 21 & 119 & 12 & 1038 & 0 & 11000 & 0 \\
\hline Goat A & 15 & 0.12 & 0.015 & 0.59 & 0 & 7 & 8 & 19 & 75 & 0 & 1400 & 0 \\
\hline Goat P & 16 & 0.12 & 0.015 & 0.59 & 0 & 7 & 8 & 19 & 75 & 0 & 1400 & 0 \\
\hline Horse & 17 & 0.034 & 0.014 & 0 & 0 & 21 & 119 & 18 & 1782 & 0 & 12000 & 0 \\
\hline
\end{tabular}

ecosystem itself. Besides, object $C$ produces objects $C^{\prime}$ which in turn generate object $C$ allowing the beginning of a new cycle. At the $\mathrm{P}$ system design, different objects (i.e. $G, G^{\prime}$ ) represent the same entity (in this case, grass) with the purpose of synchronizing the model. $T_{i}$ is an object used for counting the existing animals of species $i$. If a species overcomes the maximum density, values will be regulated. Objects $b_{0 i}, b_{i}$ and $e_{i}$ allow us to control the maximum number of animals per species in the ecosystem. At the moment when a regulation takes place, object $a_{i}$ allows us to eliminate the number of animals of species $i$ that exceeds the maximum density. Object $d_{i}$ is used to put under control domestic animals that are withdrawn from the ecosystem for their marketing.

- $\mu=\left[[]_{2}\right]_{1}$ is the membrane structure. We consider two regions, the skin and an inner membrane. The first region is important to control the densities of every species do not overcome the threshold of the ecosystem. Animals repro- 
duce, feed and die in the inner membrane. For the sake of simplicity, neutral polarization will be omitted.

- $\mathcal{M}_{1}$ and $\mathcal{M}_{2}$ are strings over $\Gamma$, describing the multisets of objects initially placed in regions of $\mu$ (encoding the initial population and the initial food);

- $\mathcal{M}_{1}=\left\{b_{0 i}, X_{i j}^{q_{i j}}, h_{t}^{q_{1, j}}: 1 \leq i \leq n, 0 \leq j \leq g_{i, 6}\right\}$, where $q_{i j}$ indicates the number of animals of species $i$ initially present in the ecosystem whose age is $j$, and $t=\max \left\{1,\left\lceil\frac{\sum_{j=8}^{21} q_{1, j}-6}{1.352}\right\rceil\right\}$;

- $\mathcal{M}_{2}=\{C\}$.

- The set $R$ of evolution rules consists of:

- The first rule represents the contribution of energetic resources to the ecosystem at the beginning of each cycle and it is essential for the system to evolve. The second rule is useful to synchronize the process.

$r_{0} \equiv\left[C \rightarrow B^{\prime \alpha} M^{\prime \beta} G^{\prime \gamma} C^{\prime}\right]_{2}^{0}$,

where $\alpha$ and $\beta$ are the double of kilos of bones and meat that are externally introduced to the ecosystem, and $\gamma$ is the amount of grass produced by the ecosystem.

$$
r_{1} \equiv\left[b_{0, i} \rightarrow b_{i}\right]_{1}^{0} .
$$

- Variation rules of the population.

We consider two cases due to the fact that in nomadic species the said variation is influenced by animals from other ecosystems.

- Case 1. Non-nomadic species $\left(k_{i, 4}=0\right)$.

- Adult males:

$$
r_{2} \equiv\left[X_{i j} \stackrel{\left(1-k_{i, 1}\right) \cdot\left(1-k_{i, 4}\right)}{\longrightarrow} Y_{i j}\right]_{1}^{0}, 1 \leq i \leq n, g_{i, 4} \leq j<g_{i, 5} .
$$

- Adult females that reproduce:

$$
\begin{aligned}
r_{3} & \equiv\left[X_{i j} \stackrel{k_{i, 2} \cdot k_{i, 1} \cdot\left(1-k_{i, 4}\right)}{\longrightarrow} Y_{i j} Y_{i 0}^{k_{i, 3}}\right]_{1}^{0}, 1 \leq i \leq 4, g_{i, 4} \leq j<g_{i, 5} . \\
r_{4} & \equiv\left[X_{i j} \stackrel{{ }_{i, 2} \cdot k_{i, 1} \cdot\left(1-k_{i, 4}\right)}{\longrightarrow} Y_{i j} Y_{i 0}^{k_{i, 3}}\right]_{1}^{0}, 7 \leq i \leq n, g_{i, 4} \leq j<g_{i, 5} . \\
r_{5} & \equiv\left[X_{5 j} \stackrel{0.5 \cdot k_{5,1}}{\longrightarrow} Y_{5 j} Y_{50}^{k_{i, 3}}\right]_{1}^{0}, g_{5,4} \leq j<g_{5,5} . \\
r_{6} & \equiv\left[X_{5 j} \stackrel{0.5 \cdot k_{5,1}}{\longrightarrow} Y_{5 j} Y_{60}^{k_{i, 3}}\right]_{1}^{0}, g_{5,4} \leq j<g_{5,5} .
\end{aligned}
$$

- Adult females that do not reproduce:

$$
r_{7} \equiv\left[X_{i j} \stackrel{\left(1-k_{i, 2}\right) \cdot k_{i, 1} \cdot\left(1-k_{i, 4}\right)}{\longrightarrow} Y_{i j}\right]_{1}^{0}, 1 \leq i \leq n, g_{i, 4} \leq j<g_{i, 5}
$$

- Old females and males that do not reproduce:

$$
r_{8} \equiv\left[X_{i j} \stackrel{1-k_{i, 4}}{\longrightarrow} Y_{i j}\right]_{1}^{0}, 1 \leq i \leq n, g_{i, 5} \leq j \leq g_{i, 6} \text {. }
$$

- Young animals that do not reproduce:

$$
r_{9} \equiv\left[X_{i j} \stackrel{1-k_{i, 4}}{\longrightarrow} Y_{i j}\right]_{1}^{0}, 1 \leq i \leq n, 1 \leq j<g_{i, 4} .
$$


- Case 2. Nomadic species $\left(k_{i, 4}=1\right)$.

$r_{10} \equiv\left[X_{1 j} h_{s} \stackrel{v_{s}}{\longrightarrow} Y_{1\left(g_{i, 4}-1\right)} Y_{1 j} h_{s+1}^{2}\right]_{1}^{0}, 1 \leq i \leq n, g_{i, 4} \leq j \leq$ $g_{i, 6}, t \leq s \leq D_{1}$, being $v_{s}=1.352 /(1.352 s+6)$ and $D_{1}=\min \{21, D+$ $t-1\}$.

$r_{11} \equiv\left[X_{1 j} h_{s} \stackrel{0.01}{\longrightarrow} Y_{1\left(g_{i, 4}-1\right)} Y_{1 j} h_{s+1}^{2}\right]_{1}^{0}, 1 \leq i \leq n, g_{i, 4} \leq j \leq$ $g_{i, 6}, D_{3} \leq s \leq D_{2}$, where $D_{2}=\max \{21, D+t-1\}$ and $D_{3}=\max \{21, t\}$.

$r_{12} \equiv\left[X_{1 j} h_{s} \stackrel{1-v_{s}}{\longrightarrow} Y_{1 j} h_{s+1}\right]_{1}^{0}, 1 \leq i \leq n, g_{i, 4} \leq j \leq g_{i, 6}, t \leq s \leq$ $D_{1}$.

$r_{13} \equiv\left[X_{1 j} h_{s} \stackrel{0.99}{\longrightarrow} Y_{1 j} h_{s+1}\right]_{1}^{0}, 1 \leq i \leq n, g_{i, 4} \leq j \leq g_{i, 6}, D_{3} \leq s \leq$ $D_{2}$.

- Mortality rules.

- Young animals that survive:

$r_{14} \equiv Y_{i j}[]_{2}^{0} \stackrel{1-m_{i, 1}-m_{i, 3}}{\longrightarrow}\left[V_{i j} T_{i}\right]_{2}^{+}, 1 \leq i \leq n, 0 \leq j<g_{i, 3}$.

- Young animals that die:

$r_{15} \equiv Y_{i j}[]_{2}^{0} \stackrel{m_{i, 1}}{\longrightarrow}\left[H_{i}^{\prime f_{i, 1} \cdot g_{i, 2}} F_{i}^{\prime f_{i, 2} \cdot g_{i, 2}} B^{\prime f_{i, 1} \cdot g_{i, 2}} M^{\prime f_{i, 2} \cdot g_{i, 2}}\right]_{2}^{+}, \quad 1 \leq i \leq$ $n, 0 \leq j<g_{i, 3}$.

- Young animals that are retired from the ecosystem:

$r_{16} \equiv\left[Y_{i j} \stackrel{m_{i, 3}}{\longrightarrow} \lambda\right]_{1}^{0}, 1 \leq i \leq n, 0 \leq j<g_{i, 3}$.

- Adult animals that do not reach an average life expectancy and survive:

$r_{17} \equiv Y_{i j} h_{s}^{k_{i, 4}}[]_{2}^{0} \stackrel{1-m_{i, 2}}{\longrightarrow}\left[V_{i j} T_{i} h_{s}^{k_{i, 4}}\right]_{2}^{+}, 1 \leq i \leq n, g_{i, 3} \leq j<g_{i, 6}, t+1 \leq$ $s \leq D+t$.

- Adult animals that do not reach an average life expectancy and die:

$r_{18} \equiv Y_{i j} h_{s}^{k_{i, 4}}[]_{2}^{0} \stackrel{m_{i, 2}}{\longrightarrow}\left[H_{i}^{\prime f_{i, 3} \cdot g_{i, 2}} F_{i}^{\prime f_{i, 4} \cdot g_{i, 2}} B^{\prime f_{i, 3} \cdot g_{i, 2}} M^{\prime f_{i, 4} \cdot g_{i, 2}} V_{i, g_{i, 4}-1}^{k_{i, 4}} h_{s}^{k_{i, 4}} T_{i}^{k_{i, 4}}\right]_{2}^{+}$, $1 \leq i \leq n, g_{i, 3} \leq j<g_{i, 6}, t+1 \leq s \leq D+t$.

- Animals that reach an average life expectancy and die in the ecosystem:

$r_{19} \equiv Y_{i g_{i, 6}} h_{s}^{k_{i, 4}}[]_{2}^{0} \stackrel{c_{20}}{\longrightarrow}\left[H_{i}^{\prime f_{i, 3} \cdot g_{i, 2}} F_{i}^{\prime f_{i, 4} \cdot g_{i, 2}} B^{\prime f_{i, 3} \cdot g_{i, 2}} M^{\prime f_{i, 4} \cdot g_{i, 2}} V_{i, g_{i, 4}-1}^{k_{i, 4}} h_{s}^{k_{i, 4}} T_{i}^{k_{i, 4}}\right]_{2}^{+}$,

$1 \leq i \leq n$, being $c_{20}=k_{i, 4}+\left(1-k_{i, 4}\right) \cdot\left(m_{i, 4}+\left(1-m_{i, 4}\right) \cdot m_{i, 2}\right), t+1 \leq$ $s \leq D+t$.

- Animals that reach an average life expectancy and are retired from the ecosystem:

$r_{20} \equiv\left[Y_{i g_{i, 6}} h_{s}^{k_{i, 4}\left(1-k_{i, 4}\right) \cdot\left(1-m_{i, 4}\right) \cdot\left(1-m_{i, 2}\right)} \lambda\right]_{1}, 1 \leq i \leq n, t+1 \leq s \leq D+t$.

- Density regulation rules. 
- Creation of objects that are going to enable the control of the maximum number of animals in the ecosystem:

$r_{21} \equiv b_{i}[]_{2}^{0} \rightarrow\left[b_{i} a_{i}^{\left\lceil 0,9 * g_{i, 7}\right]} e_{i}^{\left\lceil 0,2 * g_{i, 7}\right\rceil}\right]_{2}^{+}, 1 \leq i \leq n$.

- Evaluation of the density of the different species in the ecosystem:

$$
r_{22} \equiv\left[T_{i}^{g_{i, 7}} a_{i}^{\left(g_{i, 7}-g_{i, 8}\right)} \rightarrow \lambda\right]_{2}^{+}, 1 \leq i \leq n .
$$

- Generation of randomness in the number of animals:

$$
\begin{aligned}
& r_{23} \equiv\left[e_{i} \stackrel{0,5}{\longrightarrow} a_{i}\right]_{2}^{+}, 1 \leq i \leq n . \\
& r_{24} \equiv\left[e_{i} \stackrel{0,5}{\longrightarrow} \lambda\right]_{2}^{+}, 1 \leq i \leq n .
\end{aligned}
$$

- Change of the names of the objects which represent animals:

$$
r_{25} \equiv\left[V_{i j} \rightarrow Z_{i j}\right]_{2}^{+}, 1 \leq i \leq n, 0 \leq j<g_{i, 6} .
$$

- Change of the names of the objects which represent food resources:

$$
\begin{aligned}
r_{26} & \equiv\left[G^{\prime} \rightarrow G\right]_{2}^{+} . \\
r_{27} & \equiv\left[B^{\prime} \rightarrow B\right]_{2}^{+} . \\
r_{28} & \equiv\left[M^{\prime} \rightarrow M\right]_{2}^{+} . \\
r_{29} & \equiv\left[C^{\prime} \rightarrow C\right]_{2}^{+} . \\
r_{30} & \equiv\left[H_{i}^{\prime} \rightarrow H_{i}\right]_{2}^{+}, \quad 1 \leq i \leq n . \\
r_{31} & \equiv\left[F_{i}^{\prime} \rightarrow F_{i}\right]_{2}^{+}, \quad 1 \leq i \leq n .
\end{aligned}
$$

- Feeding rules.

$$
\begin{aligned}
& r_{32} \equiv\left[Z_{i j} h_{s}^{k_{i, 4}} a_{i} B^{f_{i, 5} \cdot g_{i, 2}} G^{f_{i, 6} \cdot g_{i, 2}} M^{f_{i, 7} \cdot g_{i, 2}}\right]_{2}^{+} \rightarrow X_{i(j+1)} h_{s}^{k_{1,4}}[]_{2}^{0}, \quad 1 \leq \\
& i \leq n, 0 \leq j \leq g_{i, 6}, \quad t+1 \leq s \leq D+t .
\end{aligned}
$$

- Updating rules.

The purpose of the following rules is to make a balance at the end of the year. That is, the leftover food is not useful for the next year, so it is necessary to eliminate it. But if the amount of food is not enough, some animals die.

- Elimination of the remaining bones, meat and grass:

$$
\begin{aligned}
r_{33} & \equiv[G \rightarrow \lambda]_{2}^{0} . \\
r_{34} & \equiv[M \rightarrow \lambda]_{2}^{0} . \\
r_{35} & \equiv[B \rightarrow \lambda]_{2}^{0} . \\
r_{36} & \equiv\left[T_{i} \rightarrow \lambda\right]_{2}^{0}, \quad 1 \leq i \leq n . \\
r_{37} & \equiv\left[a_{i} \rightarrow \lambda\right]_{2}^{0}, \quad 1 \leq i \leq n . \\
r_{38} & \equiv\left[e_{i} \rightarrow \lambda\right]_{2}^{0}, \quad 1 \leq i \leq n .
\end{aligned}
$$




$$
\begin{aligned}
r_{39} & \equiv\left[b_{i}\right]_{2}^{0} \rightarrow b_{i}[]_{2}^{0}, 1 \leq i \leq n . \\
r_{40} & \equiv\left[H_{i}\right]_{2}^{0} \rightarrow H_{i}[]_{2}^{0}, 1 \leq i \leq n . \\
r_{41} & \equiv\left[F_{i}\right]_{2}^{0} \rightarrow F_{i}[]_{2}^{0}, 1 \leq i \leq n .
\end{aligned}
$$

- Young animals that die because of a lack of food:

$$
\begin{aligned}
r_{42} & \equiv\left[Z_{i j} \stackrel{g_{i, 1}}{\longrightarrow} H_{i}^{\prime f_{i, 1}} F_{i}^{\prime f_{i, 2}} B^{\prime f_{i, 1}} M^{\prime f_{i, 2}}\right]_{2}^{0}, 1 \leq i \leq n, 0 \leq j<g_{i, 3} . \\
r_{43} & \equiv\left[Z_{i j}\right]_{2}^{0} \stackrel{1-g_{i, 1}}{\longrightarrow} d_{i}[]_{2}^{0}, 1 \leq i \leq n, 0 \leq j<g_{i, 3} .
\end{aligned}
$$

- Adult animals that die because of a lack of food:

$$
\begin{aligned}
& r_{44} \equiv\left[Z_{i j} h_{s}^{k_{1,4}} \stackrel{g_{i, 1}}{\longrightarrow} H_{i}^{\prime f_{i, 3}} F_{i}^{\prime f_{i, 4}} B^{\prime f_{i, 3}} M^{\prime f_{i, 4}}\right]_{2}^{0}, 1 \leq i \leq n, g_{i, 3} \leq j \leq \\
& g_{i, 6}, t+1 \leq s \leq D+t . \\
& r_{45} \equiv\left[Z_{i j} h_{s}^{k_{1,4} \stackrel{1-g_{i, 1}}{\longrightarrow}} \lambda\right]_{2}^{0}, 1 \leq i \leq n, g_{i, 3} \leq j \leq g_{i, 6}, t+1 \leq s \leq \\
& D+t .
\end{aligned}
$$

The purpose of these rules is to eliminate objects $\mathrm{H}$ and $\mathrm{F}$ associated with the quantity of biomass left by every species.

$$
\begin{aligned}
r_{46} & \equiv\left[H_{i} \rightarrow \lambda\right]_{1}^{0}, 1 \leq i \leq n . \\
r_{47} & \equiv\left[F_{i} \rightarrow \lambda\right]_{1}^{0}, 1 \leq i \leq n .
\end{aligned}
$$

\subsection{Structure of the P System Running}

The model of the ecosystem presented in the previous Section includes new ingredients with the aim to overcome the limitations found at the model described in [1]. More specifically, the modifications made are the following:

- It has been added new species which have active roles in the ecosystem under study, although their roles are perhaps less relevant that those of the first species studied. These species are the ovis orientalis, the wild boar, the horse, the goat and the cow. Besides, it has been included greedy species such as the Egyptian Vulture and the Griffon Vulture which compete with the Bearded Vulture.

- It is considered that the population growth rate of the Bearded Vulture varies depending on the surface and orography of the system as well as on the existing population.

- A new module has been added in order to regulate the population density of the ecosystem.

- The mortality module has been modified in order to consider that after an animal dies, in addition to the bones it leaves at the ecosystem, its meat serves as food for other animals. 
- The feeding module has also been modified because the feeding resources for the species at the ecosystem have been modelled in this new approach. For this reason, new objects have been introduced representing, apart from the bones, the amount of meat and grass available at the ecosystem.

In this model, a module devoted to control the density has been introduced. From the point of view of the execution of the system, the module has been incorporated between the Mortality and the Feeding modules. These are depicted in Figure 1.

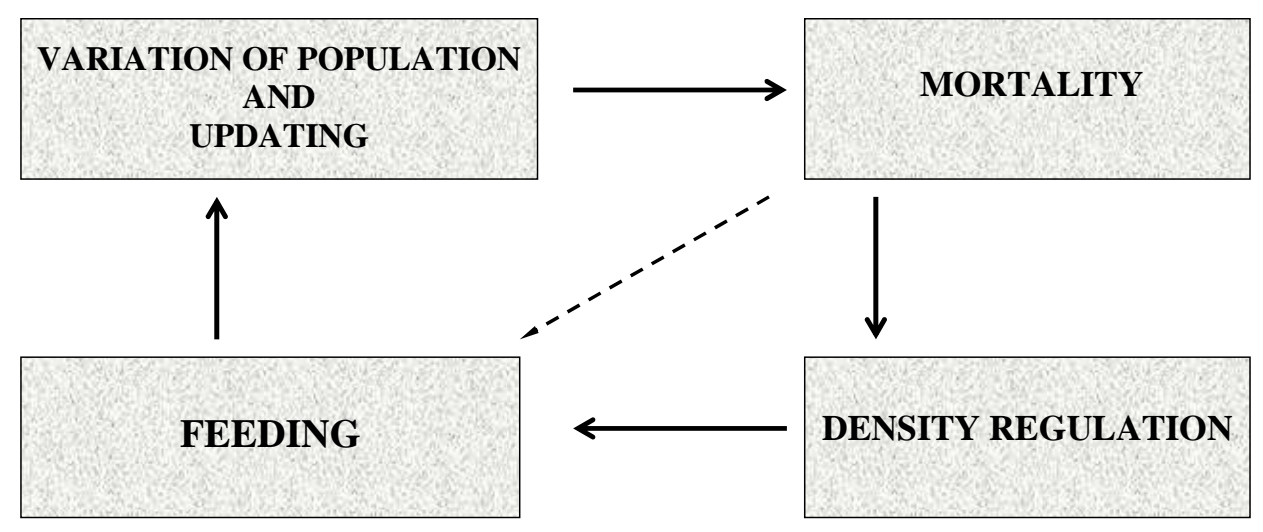

Fig. 1. Modules of the P system

Let us recall that, objects $X$ represent the different species along the execution of the reproduction module. Objects $X$ evolve to objects $Y$ (mortality module) when they pass to the mortality module, and these objects $Y$ evolve to objects $V$ (density module), together with objects $T$ which represent the number of individuals per each species. Then, objects $V$ evolve to objects $Z$ (feeding module). Objects $T$ will allow the activation of the process of auto-regulation of the ecosystem when the number of individuals of a species exceed the threshold of maximum density, which is codified by objects $a$.

When a cycle is produced, all objects which are not associated with species are eliminated, except the biomass generated by the animals that have died due to the process of regulation.

\section{Results and Discussions}

The software tool used for the purposes of this paper is based on P-Lingua 2.0 [4]. P-Lingua is a new programming language able to define $\mathrm{P}$ systems of different 
types (from now on, frameworks). For instance, P-Lingua can define any P system within the probabilistic framework mentioned in this paper.

Next, we describe how to implement in $\mathrm{P}$-Lingua the applicability of the rules to a given configuration.

(a) Rules are classified into sets so that all the rules belonging to the same set have the same left-hand side.

(b) Let $\left\{r_{1}, \ldots, r_{t}\right\}$ be one of the said sets of rules. Let us suppose that the common left-hand side is $u[v]_{i}^{\alpha}$ and their respective probabilistic constants are $c_{r_{1}}, \ldots, c_{r_{t}}$. In order to determine how these rules are applied to a give configuration, we proceed as follows:

- It is computed the greatest number $\mathrm{N}$ so that $u^{N}$ appears in the father membrane of $i$ and $v^{N}$ appears in membrane $i$.

- N random numbers $x$ such that $0 \leq x<1$ are generated.

- For each $k(1 \leq k \leq t)$ let $n_{k}$ be the amount of numbers generated belonging to interval $\left[\sum_{j=0}^{k-1} c_{r_{j}}, \sum_{j=0}^{k} c_{r_{j}}\right.$ ) (assuming that $c_{r_{0}}=0$ ).

- For each $k(1 \leq k \leq t)$, rule $r_{k}$ is applied $n_{k}$ times.

P-Lingua 2.0 provides a JAVA library that defines algorithms in order to simulate $\mathrm{P}$ system computations for each supported framework, so we are using a common algorithm for all $\mathrm{P}$ systems within the probabilistic framework.

By defining the ecosystem model by a $\mathrm{P}$ system written in P-Lingua, it is possible to check, validate and improve the model in a flexible way, instead of developing a new "ad hoc" simulator for each new model.

The application has a friendly user-interface, which sits on the P-Lingua JAVA library, allowing the user to change the initial parameters of the ecosystem in an easy way without special knowledge about the $\mathrm{P}$ system or the initial multisets. The main objetive is to make virtual experiments on the ecosystem.

The current version of this software is a prototype GPL licensed [8].

The model designed is experimentally validated by using the simulator previously described as well as the data from Table 2 .

\begin{tabular}{|l|c|c|c|c|c|c|c|c|c|c|c|c|}
\hline Specie & $\mathbf{7 9}$ & $\mathbf{8 4}$ & $\mathbf{8 7}$ & $\mathbf{8 9}$ & $\mathbf{9 3}$ & $\mathbf{9 4}$ & $\mathbf{9 5}$ & $\mathbf{9 9}$ & $\mathbf{0 0}$ & $\mathbf{0 5}$ & $\mathbf{0 8}$ & $\mathbf{0 9}$ \\
\hline Bearded V. & - & 7 & - & 13 & - & - & 21 & - & 28 & 34 & 35 & - \\
\hline Egyptian V. & - & - & 29 & - & 34 & - & - & - & 40 & - & 66 & - \\
\hline Griffon V. & 38 & 118 & - & - & - & - & - & 431 & - & - & - & 1125 \\
\hline Pyrenean C. & - & - & - & - & - & 9000 & - & - & - & - & 12000 & - \\
\hline Red deer & - & - & - & - & - & 1000 & - & - & - & - & 5500 & - \\
\hline Fallow deer & - & - & - & - & - & 600 & - & - & - & - & 1500 & - \\
\hline Roe deer & - & - & - & - & - & 1000 & - & - & - & - & 10000 & - \\
\hline
\end{tabular}

Table 2. Number of animals in the Catalan Pyrenees (1979-2009)

At the validation process, we have focused on the evolution of savage species populations. For that purpose, it has been validated the ecosystem dynamics for 
a period of 14 years, since 1994. The Bearded Vulture (respectively the Griffon and the Egyptian Vultures) populations at the initial year has been considered according to the data Table 2 by means of a logarithmic (respectively, exponential) regression (see Figure 2).

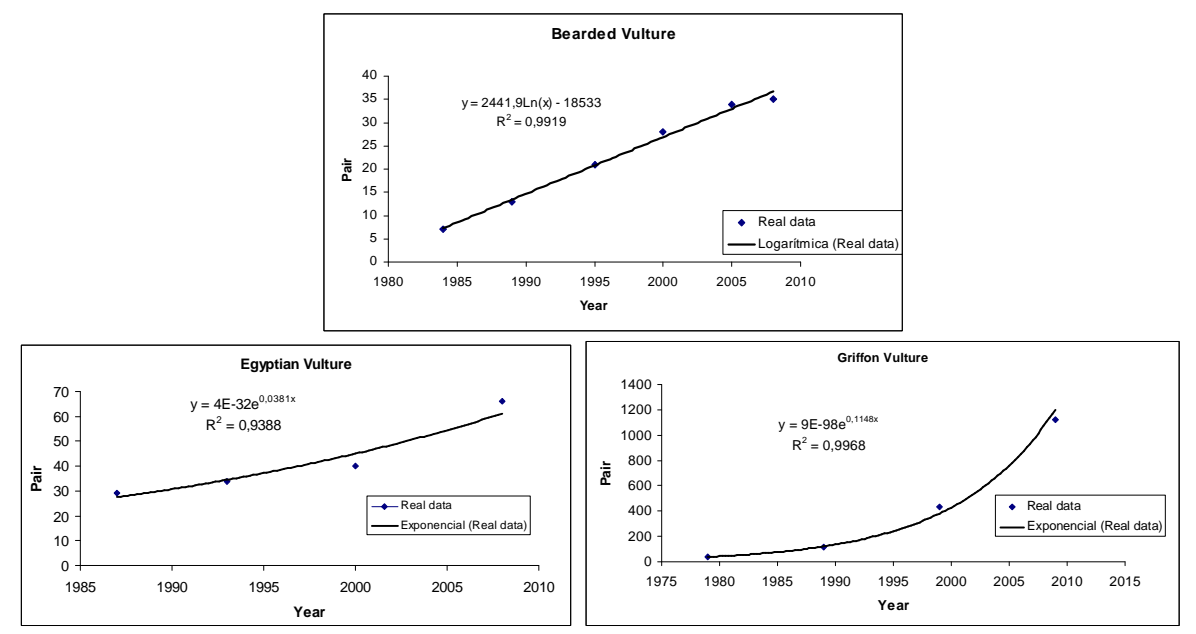

Fig. 2 Regression relationships between numbers of pairs and years

At the validation process, values obtained from the simulator running have been compared to those obtained experimentally. It is important to remember that we have focused on the population dynamics at savages species from which there are only data about the initial (1994) and final (2008) years, except for scavengers birds which we have more information about (see Table 2 for details).

Bearing in mind the model designed is probabilistic, the ecosystem evolution throughout the period under study has been obtain by running the simulator for 100 times having the same input data. The simulator executions have allowed us to estimate the standard deviation and compute the population confidence intervals of the different species. The result presented in Figure 3 is the average of the 100 simulator executions.

Finally, we have compared the model presented in this work (we refer to it as model II) to the model presented in [1] (we refer to it as model I). For that purpose, we have used the simulator previously described studying the ecosystem evolution for a period of 10 years from 1994 on. Some of the results are shown at the Figure 4. Both models present good results until 2008, regarding experimental data (except for the Pyrenees Chamois. Nonetheless, at the simulations corresponding to the years later to 2008, it is noticed a great difference between models due to the fact that model I did not consider the regulation of the populations. 

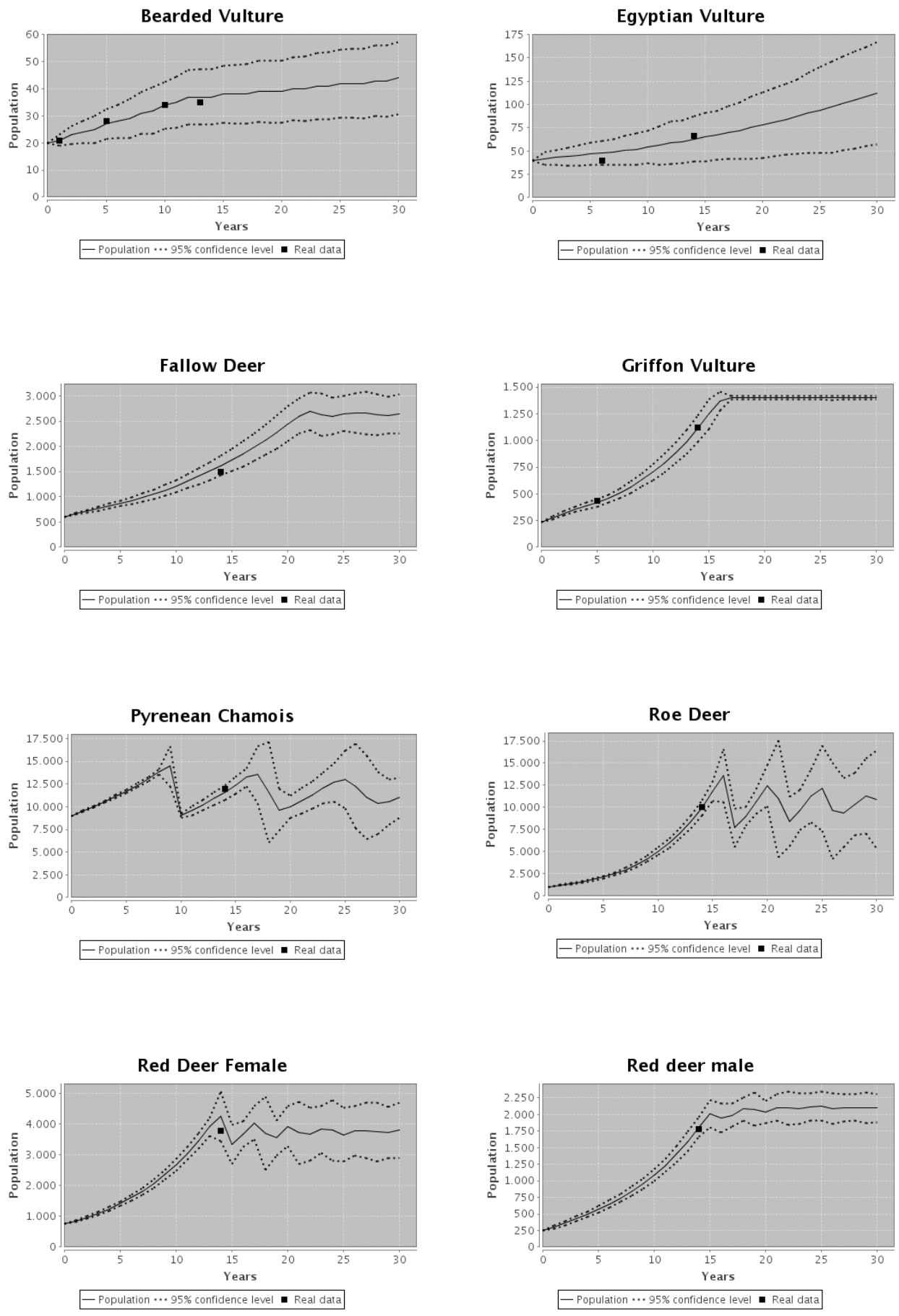

Fig. 3 Experimental Validation 

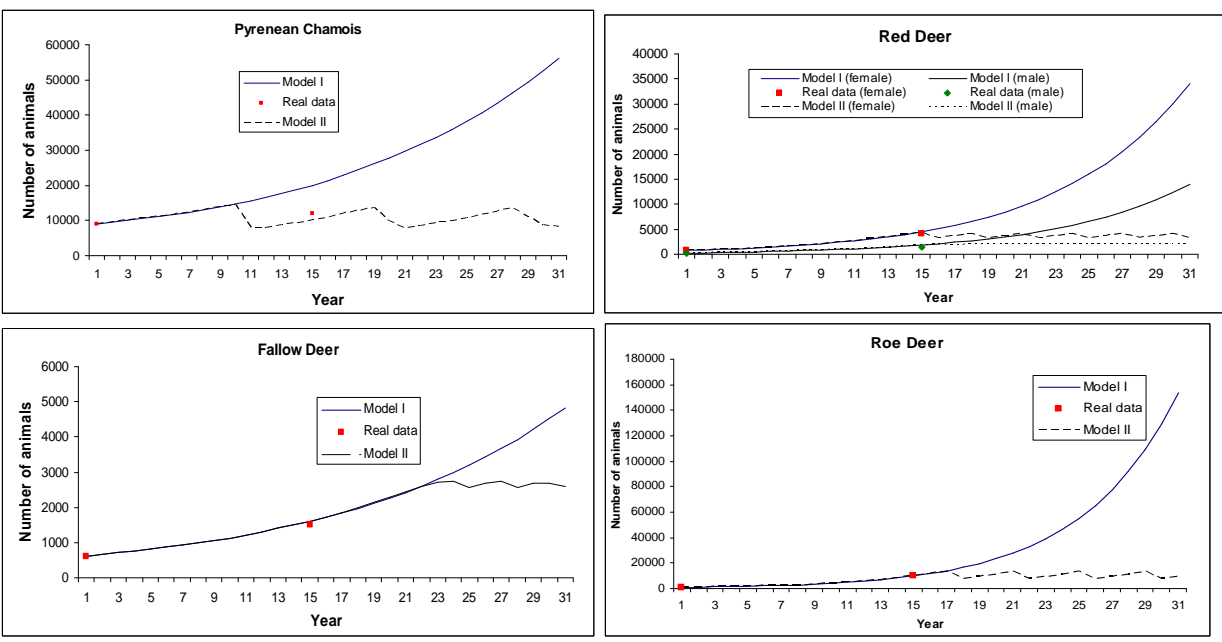

Fig. 4 Result of both models

\section{Conclusions and Future Works}

At [1], it was presented a model of an ecosystem related to the Bearded Vulture at the Catalan Pyrenees. The said model was based on a probabilistic P system which included 5 species, did only consider the amount of food available for the Bearded Vulture and did not consider the maximum number of animals that can coexist in the ecosystem.

In this paper, a new model of the said ecosystem has been designed. This model considers 13 species, including two new types of scavenger birds, autoregulation, the energetic needs of all the species and the fact that the Bearded Vulture population may not have a constant growth.

Nonetheless, we have considered some important restrictions at the design of the new model. More specifically, we have assumed a uniform distribution of the different species and population increases and decreases due to the fact that the external flow of the ecosystem have not been considered.

A new simulator written in JAVA which uses the specification language P Lingua [4] has been used to experimentally validate the model designed. The said simulator has also been used in order to compare the results presented in this paper with to those presented in [1]. This new simulator allows us to modify the different parameters of the $\mathrm{P}$ system (constants associated with rules and the initial multisets) in order to study the ecosystem dynamics and the different initial condition. In this way, once the model is considered to be experimentally validated, it is possible to carry out virtual experiments in the system which can provide hypotheses about the possible evolution of the ecosystem. These hypotheses, filtered 
by experts in a suitable way, can be useful for the ecologists when taking decisions which favour both the balance of the ecosystem and the preservation of the endangered species such as the Bearded Vulture.

In a future work, we hope to add new ingredients to this model which overcome the restrictions imposed on it that where previously referred to. For that purpose, we are studying the possibility of considering multienvironment $\mathrm{P}$ systems (see [7], for details) as a new modelling scenario. This will imply an important revision of the simulator and the searching of more efficient algorithms which simulate the running of the the probabilistic strategy.

\section{Acknowledgement}

The authors acknowledge the support of the project TIN2006-13425 of the Ministerio de Educación y Ciencia of Spain, cofinanced by FEDER funds, and the support of the Project of Excellence with Investigador de Reconocida Valia of the Junta de Andalucía, grant P08-TIC-04200.

\section{References}

1. M. Cardona, M. A. Colomer, M.J. Pérez-Jiménez, D. Sanuy, A. Margalida. Modelling ecosystems using P Systems: The Bearded Vulture, a case of study. Lecture Notes in Computer Science, 5391 (2009), 137-156.

2. C.J.Brown. Population dynamics of the Bearded Vulture Gypaetus barbatus in southern Africa. African Journal of Ecology, 35 (1997), 53-63.

3. J.A. Donázar. Los buitres ibéricos: biología y conservación. J.M. Reyero Editor, Madrid, Spain, 1993.

4. M. García-Quismondo, R. Gutiérrez-Escudero, M.A. Martnez, E. Orejuela, I. PérezHurtado. P-Lingua 2.0: A sofware framework for cell-like P systems. International Journal of Computers, Comunications and Control, Vol. IV, 3 (2009), 234-243.

5. A. Margalida, J.Bertran, R. Heredia: Diet and food preferences of the endangered Bearded Vulture Gypaetus barbatus: a basis for their conservation. Ibis 151 (2009), 235-243.

6. A. Margalida, D. García, A. Cortés-Avizanda. Factors influencing the breeding density of Bearded Vultures, Egyptian Vultures and Eurasian Griffon Vultures in Catalonia (NE Spain): management implications. Animal Biodiversity and Conservation, 30, 2 (2007), 189-200.

7. F.J. Romero, M.J. Pérez-Jiménez. A model of the Quorum Sensing System in Vibrio Fischeri using P systems. Artificial Life, 14, 1 (2008), 95-109.

8. GPL license: http://www.gnu.org/copyleft/gpl.html 